\title{
Mapping electrostatic potential around a Pt nanoparticle supported on $\mathrm{TiO}_{2}$ (110)
}

Yoshio Takahashi ${ }^{1}$, Tetsuya Akashi ${ }^{1}$, Hajime Hojo ${ }^{2}$, Hisahiro Einaga ${ }^{2}$, Hiroshi Nakajima ${ }^{3}$, Toshiaki Tanigaki $^{4}$, Hiroyuki Shinada ${ }^{1}$ and Yasukazu Murakami ${ }^{5}$

${ }^{1}$ Research \& Development Group, Hitachi, Ltd., Hatoyama, Saitama, Japan, ${ }^{2}$ Department of Materials Science, Faculty of Engineering Sciences, Kyushu University, Fukuoka, Japan, ${ }^{3}$ Department of Materials Science, Osaka Prefecture University, Sakai, Osaka, Japan, ${ }^{4}$ Research \& Development Group, Hitachi, Ltd., Saitama, Japan, ${ }^{5}$ Department of Applied Quantum Physics and Nuclear Engineering, Kyushu University, Fukuoka, Japan

Platinum (Pt) nanoparticles supported on a titanium oxide (TiO2) crystal have high catalytic activities for selective hydrogenation reactions in producing energy and environmental materials. Although a lot of research has been conducted in the last few decades on characterizing Pt-TiO2 systems, the electrostatic potential around $\mathrm{Pt}$ nanoparticles has not been reported yet. In this presentation, we show the weak electrostatic fields of a $\mathrm{Pt} / \mathrm{TiO} 2$ catalyst successfully observed using electron holography, which is a phase detection method using the interference between the object electron waves and the reference waves traveling in a vacuum at the nanometer scale region. The microscope we used was a 1.2-MV atomic resolution holography electron microscope developed in the FIRST project[1], which was equipped with a cold field emission electron gun, the CEOS spherical aberration corrector, double biprism interferometers, and a Gatan K2 summit CMOS direct detection camera. The specimen we studied was made using a photochemical reduction of hexachloroplatinic acid on an ion-milled and annealed rutile substrate. We observed Pt particles (5-10 $\mathrm{nm}$ in diameter) attached on a substrate edge with a (110) crystal facet. The specimen was set in a vacuum of the microscope after plasma cleaning and holography observations were carried out at room temperature. The projected electrostatic potential in vacuum is proportional to the phase shift of the object waves in electron holography. For example, if a Pt nanoparticle with a diameter of $5 \mathrm{~nm}$ has $0.2 \mathrm{~V}$ surface potential, the phase shift amount near the edge of the Pt particle is expected to be $\sim 2 \pi / 1000 \mathrm{rad}$. Therefore, we need a high-precision and high-accuracy phase detection method. The precision of phase detection can be improved by increasing the number of illumination electrons. To achieve this, hundreds of holograms were collected, and the reconstructed phase images were averaged a posteriori. Because the phase images were acquired at atomic resolution, the images were aligned at atomic resolution using their crystal lattice patterns with an automated template matching method. The accuracy of the phase detection could be improved by subtracting reference phase images of the vacuum area far from the object. The reference image contains the distortions of projection lenses, the charging effect at biprisms, and the variation of the camera pixel sensitivity. However, the background phase distortion could have been changed if the reference holograms had been acquired after collecting hundreds of object holograms. Actually, our off-line test without a sample showed $2 \pi / 1000 \mathrm{rad}$ level distortion appeared at $\sim 15$ minutes later. To avoid this problem, object holograms and reference holograms were divided into a small number of groups and were collected in their respective groups. Figure 1(a) shows a phase image of a Pt nanoparticle on a $\mathrm{TiO} 2$ (110) substrate, which was constructed by averaging phase images from 160 holograms of the object. The background noise level of the averaged phase image is less than $2 \pi / 850 \mathrm{rad}$ (standard deviation). Figure 1(b) shows a line profile near the Pt nanoparticle. A phase increase of about $2 \pi / 220 \mathrm{rad}$ in the vacuum at the top of Pt was observed. The electrostatic potential near the Pt nanoparticle appears to have a positive potential generated by an electrostatic dipole of $\sim 300$ $\mathrm{D}$ (eight electron charges with a distance of $0.8 \mathrm{~nm}$ ) located at a boundary between the $\mathrm{Pt}$ and $\mathrm{TiO} 2$ surfaces. We will discuss the origin of the electrostatic potential by comparing the phase distribution of 
other particles and the variations over time. In conclusion, phase shifts around a Pt nanoparticle supported on a $\mathrm{TiO} 2$ substrate were observed at atomic resolution by collecting many holograms and by averaging the aligned phase images. We demonstrated the detection of a weak electrostatic potential appearing in catalytic material .
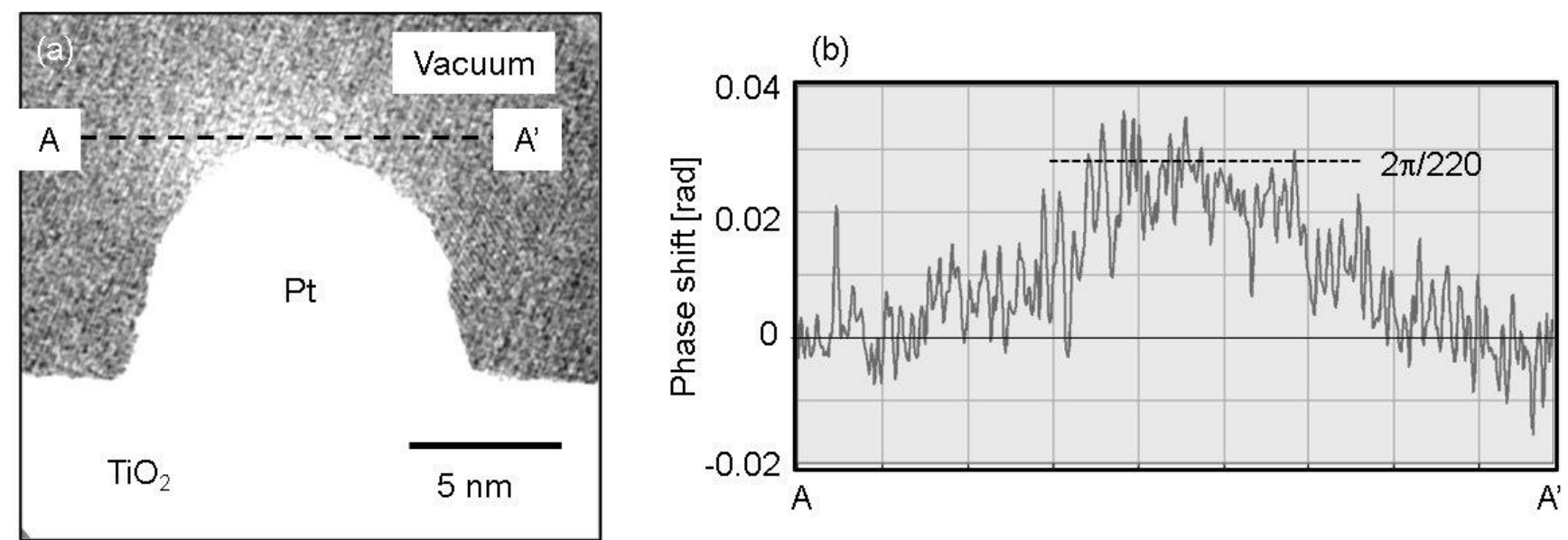

Figure 1. (a) Phase map around a Pt nanoparticle on a TiO2 (110) substrate. The brightness is adjusted to a range of $-2 \pi / 200$ to $2 \pi / 200 \mathrm{rad}$. (b) Line profile along dashed line $\mathrm{AA}^{\prime}$ ' in (a). The positive electrostatic potential was detected in a vacuum near the top surface of the Pt particle.

\section{References}

[1] T. Akashi et al., Appl. Phys. Lett., 106 (2015) 074101.

[2] This work was supported by JST CREST Grant Number JPMJCR 1664, Japan. 